\title{
CONVERGENCIA DEL CRECIMIENTO ECONÓMICO Y DESIGUALDAD ENTRE PAÍSES DE CENTROAMÉRICA (1962-2012)
}

\author{
CONVERGENCE OF ECONOMIC GROWTH AND INEQUALITY BETWEEN CENTRAL \\ AMERICAN COUNTRIES (1962-2012)
}

\section{Marianela Molina Hernández ${ }^{1}$ Marco Vinicio Valerio Berrocal ${ }^{2}$}

\section{Resumen}

En este documento se estudia la hipótesis de convergencia en el producto interno bruto (PIB) per cápita en los países centroamericanos durante el periodo 1962 hasta el 2012.

Se determinó que la velocidad de convergencia entre los países de Centroamérica es lenta $(\beta=-0,09597)$ y tiende a desacelerarse a lo largo del tiempo. Además la evidencia apunta a que la convergencia es en diferentes estados estacionarios, y que esta es muy fuerte en las tasas de crecimiento.

Además, se estudia la relación existente entre crecimiento económico y la desigualdad en el ingreso (coeficiente de GINI), y se determina que existe un trade off ${ }^{3}$ entre crecimiento y desigualdad.

Palabras clave: Convergencia; Datos de Panel; Desigualdad; Crecimiento Económico.

doi: http://dx.doi.org/10.15359/eys.20-47.1

Fecha de recepción: 5 de noviembre del 2014. Fechas de reenvíos: 15 de abril del 2015, 28 de abril del 2015. Fecha de aceptación 9 de junio del 2015. Fecha de publicación 15 de junio del 2015

${ }^{1}$ Bachiller en Economía. Universidad Nacional, Costa Rica, mari molh@ @otmail.com

2 Bachiller en Economía. Universidad Nacional, Costa Rica, markxsw@gmail.com

${ }^{3}$ Por trade off se da a entender una situación donde hay que renunciar a una característica para obtener otra.

Marianela Molina Hernández y Marco Vinicio Valerio Berrocal

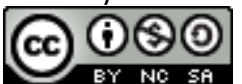

Revista Economía y Sociedad by Universidad Nacional is licensed under a Creative Commons Reconocimiento-NoComercial- 


\begin{abstract}
The growth convergence hypothesis of the per capita Gross Domestic Product (GDP) in Central American countries during the 1962 - 2012 period is discussed in this paper.
\end{abstract}

It was determined that the rate of convergence between the Central American countries is slow $(\beta=-0.09597)$ and tends to decrease with time. In addition, evidence suggests that convergence is in different steady states and is strong in the GDP growth rate.

The paper also analyzes the relationship between economic growth and income inequality (Gini Index) and determines the existence of a tradeoff between growth and inequality.

Keywords: Convergence; Panel Data; Income Inequality; Economic Growth.

\title{
Introducción
}

El crecimiento económico se deriva del aumento en la cantidad producida de bienes y servicios, esto se traduce en el aumento de la renta nacional. Esta suele medirse en renta per cápita para lograr captar el aumento de la población ${ }^{4}$; para que un país pueda lograr el crecimiento de la renta per cápita debe contar con mano de obra capacitada y con tecnología avanzada, a su vez debe lograr también aumentar el poder adquisitivo de sus habitantes para poder impulsar el crecimiento.

La desigualdad se resume en las disparidades de ingresos que hay entre distintos habitantes que los hace capaces de poseer más o menos bienes materiales, la mayoría de los gobiernos se preocupan porque los países tengan niveles mínimos de desigualdad, sin embargo esto no suele ocurrir, alrededor del mundo los países tienen altos niveles de desigualdad tanto a lo interno de los países como regionalmente.

La convergencia del crecimiento y la reducción de la desigualdad suele estar cercana a alcanzarse en los países más desarrollados. En los menos desarrollados esto es algo un tanto más difícil de lograr debido a que no siempre se encuentran las condiciones necesarias para el crecimiento, o bien existen altos niveles de pobreza y desempleo, lo que dificulta la ruta al desarrollo; y tal es el caso de los países centroamericanos.

\footnotetext{
${ }^{4}$ El ingreso per cápita como indicador de bienestar se fundamenta desde la lógica utilitarista donde $U=U(x 1, x 2, x n)$, donde xn es igual a una cantidad de bienes o servicios, la principal crítica a este indicador es que no contempla externalidades, el medio ambiente o la distribución del ingreso; es decir, responde a la visión neoclásica de desarrollo.
}

2

Marianela Molina Hernández y Marco Vinicio Valerio Berrocal

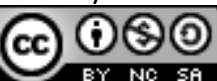

Revista Economía y Sociedad by Universidad Nacional is licensed under a Creative Commons Reconocimiento-NoComercialCompartirlgual 4.0 Internacional License. Creado a partir de la obra en http://www.revistas.una.ac.cr/index.php/economia 
Centroamérica se caracteriza por ser una región muy desigual, pero a pesar de esto la integración regional es una prioridad para distintas autoridades de política ${ }^{5}$, Kumo (2011) coincide con que una fuerte convergencia en el crecimiento es una condición necesaria para la integración regional. En este contexto que el estudio de la convergencia del crecimiento económico cobra relevancia. La movilidad de factores de producción y el comercio de bienes tiene un rol fundamental en la convergencia y no solo en los niveles de crecimiento, sino también es de esperarse que otros indicadores como la desigualdad del ingreso tienda a converger en el tiempo en un valor único de equilibrio.

Esta investigación estima la convergencia del crecimiento entre países centroamericanos y su relación con la desigualdad del ingreso mediante una aproximación de datos de panel que abarca los años 1962 al 2012.

El documento se divide de la siguiente manera: en la segunda sección se realiza una síntesis de la teoría de la convergencia del crecimiento, en una tercera sección se presenta la metodología, así como los modelos teóricos que serán estimados y las hipótesis que en estos subyacen. Por último, se presentan los resultados y conclusiones del estudio.

\section{Consideraciones teóricas}

La principal definición de convergencia usada en la literatura moderna se basa en la relación entre ingreso inicial y el crecimiento posterior.

\subsection{Convergencia absoluta $($ beta $=\boldsymbol{\beta})$}

El concepto de convergencia beta $(\boldsymbol{\beta})$ se ha desarrollado sobre la base de los modelos de crecimiento neoclásicos. Estos modelos de crecimiento conducen al establecimiento de una relación inversa entre la tasa de crecimiento de la renta por habitante (o el producto por empleado) y el nivel inicial de dicha renta (productividad).

Según la teoría neoclásica, una economía converge hacia el estado de equilibrio, en el que la producción es constante y la tasa de crecimiento es cero, debido a los rendimientos decrecientes de la inversión en capital fijo. A lo largo de la transición de la economía hacia el estado estacionario, la tasa de crecimiento es inversamente proporcional a la relación capital-trabajo, es decir, cuanto menor sea la relación capital-trabajo, mayor será la tasa de crecimiento. Así, los países con menor capital per cápita inicial tienden a crecer más rápido en comparación con aquellos países con mayor capital per cápita inicial. En otras palabras, si

5 Existen diversos esfuerzos de integración no solo de Centroamérica, sino también a nivel latinoamericano. Algunos de los tratados que demuestran estos esfuerzos es el Mercado Común Centroamericano, la Alianza del Pacifico, etc. Además, existe todo un marco institucional para la integración como es el Sistema de la Integración Centroamericana (SICA).

Marianela Molina Hernández y Marco Vinicio Valerio Berrocal 
se presentan rendimientos decrecientes de capital, una economía con menor relación capital-trabajo exhibe un producto marginal del capital mayor y, por tanto, crece más rápido comparada con otra economía similar con mayor relación capital-trabajo, donde las diferencias entre los países tienden a desaparecer con el tiempo, con el ingreso per cápita y su tasa de crecimiento converge gradualmente hasta alcanzar un equilibrio a largo plazo idéntico para ambos países, respectivamente.

Según Antunez (2011), a largo plazo los países del mundo que solo difieran en su relación capital-trabajo tenderán a un mismo estado de crecimiento proporcionado. En este sentido, aquellas economías que se encontraban en una situación menos favorable (nivel de ingreso per cápita menor), mostrarán una propensión a tasas de crecimiento superiores a las economías más desarrolladas (nivel de ingreso per cápita superior).

Así, Barro y Sala-i-Martin (1990) utilizan la siguiente expresión para contrastar la hipótesis de convergencia beta a partir de la log-linearización del modelo neoclásico con tecnología CobbDouglas y suponen que tanto el progreso técnico como la tasa de ahorro se determinan de manera exógena:

$$
\text { (1) }\left(\frac{1}{\mathrm{~T}}\right) \log \left(\frac{\mathrm{y}_{\mathrm{it}}}{\mathrm{y}_{\mathrm{i}, \mathrm{t}-\mathrm{T}}}\right)=\mathrm{a}+\left(\frac{1-\mathrm{e}^{-\beta \mathrm{T}}}{\mathrm{T}}\right) \log \left(\mathrm{y}_{\mathrm{i}, \mathrm{t}-\mathrm{T}}\right)+\mathrm{u}_{\mathrm{it}, \mathrm{t}-\mathrm{T}}
$$

Donde $\beta=(1-a)(x+n+\delta)$ denota la velocidad de convergencia hacia el estado estacionario del sistema ( $\alpha$ es el coeficiente asociado con el capital en la función de producción agregada, $x$ es la tasa de crecimiento del progreso técnico que se ha supuesto exógena, $\mathrm{n}$ refleja la tasa de crecimiento de la población mientras que, por último, $\delta$ es la tasa de depreciación). El término constante es tal que:

$$
\text { (2) } \quad \alpha=x+\left(\frac{1-\mathrm{e}^{-\beta \mathrm{T}}}{\mathrm{T}}\right) \log (\mathrm{y} *)
$$

Con $\mathrm{y}^{*}$ como el valor de y correspondiente al estado estacionario y que se supone común para todas las regiones (convergencia beta absoluta).

Se dirá que existe convergencia si el coeficiente $\alpha$ de esta ecuación es afectado por un signo negativo, lo que implica una relación negativa entre la tasa de crecimiento promedio durante el periodo de estudio y el logaritmo del ingreso per cápita inicial.

\subsection{Convergencia $\beta$ condicional}

La convergencia condicional permite que cada país tenga un nivel diferente de ingreso per cápita hacia el cual está convergiendo. Esto implica que cada país converge hacia su propio estado estacionario y que a largo plazo todas las tasas de crecimiento serán igualadas.

4

Marianela Molina Hernández y Marco Vinicio Valerio Berrocal

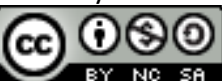

Revista Economía y Sociedad by Universidad Nacional is licensed under a Creative Commons Reconocimiento-NoComercialCompartirlgual 4.0 Internacional License. Creado a partir de la obra en http://www.revistas.una.ac.cr/index.php/economia 
Asimismo, se plantea que cada grupo de países tiende a largo plazo, a su propio estado de crecimiento proporcionado. Además de que aquellos países que al inicio tenían relativamente un menor capital por trabajador, crecerán dentro de su propio grupo más rápido que los otros países que al inicio poseían más capital por trabajador.

El modelo neoclásico de crecimiento también predice que debido a diferencias en los factores que determinan la senda de crecimiento económico en diferentes países, es posible que una economía presente un ingreso inicial alto y un crecimiento rápido simplemente porque su actual ingreso es mucho menor que su ingreso en estado estacionario. Por ejemplo, valores más altos para el cambio tecnológico en la ecuación de estado estacionario tienden a generar alta relación capital-trabajo y altos ingresos en estado estacionario.

Los dos tipos de convergencia $\beta$ tienen implicaciones muy distintas. La convergencia $\beta$ absoluta dentro de un grupo de economías implica una tendencia a la igualación de las rentas per cápita. A largo plazo, el nivel esperado de renta per cápita es el mismo para todos los miembros del grupo, independientemente de su valor inicial. Esto no quiere decir, por supuesto, que la desigualdad llegue a desaparecer por completo, puesto que siempre habrá perturbaciones con efectos desiguales en distintos territorios. Sin embargo, tales perturbaciones tendrán tan solo efectos transitorios, lo que implica que a largo plazo debería observarse una distribución fluida en la que los países o regiones cambian su posición relativa con bastante frecuencia.

La convergencia $\beta$ condicional, con diferentes estados estacionarios, daría lugar a una situación muy diferente. Aunque cada territorio mostrará una tendencia a converger a su propio estado estacionario, estos podrían ser muy distintos entre sí. Por lo tanto, pueden persistir disparidades importantes en las posiciones relativas de los mismos.

\subsection{Convergencia relativa (sigma)}

El concepto de sigma convergencia contribuye a la medición de la convergencia con alguna clase de información acerca de la dinámica de la distribución del ingreso entre los países. De acuerdo con el contraste de $\beta$-convergencia, esta se produce cuando la dispersión entre los niveles de renta de distintos países disminuye con el tiempo.

La convergencia absoluta y la relativa están relacionadas entre sí, pero distan mucho de ser equivalentes. La existencia de algún tipo de convergencia absoluta es una condición necesaria, pero no suficiente para la convergencia relativa.

\section{Metodología y datos}

Se utilizaron regresiones de datos de panel para estimar la velocidad de convergencia del crecimiento en los países centroamericanos. Se realizan las estimaciones mediantes el estimador 
de efectos fijos según lo propuesto por Baltagi $(2005)^{6}$, y se emplean errores estándar robustos ${ }^{7}$. Este método de estimación se adopta para estimar diferentes elasticidades que ayudarán a explicar las relaciones de variables como educación, inversión pública, etc. La estimación de estas elasticidades servirá como base para el análisis de políticas de forma contra factual, y ayudarán a caracterizar la naturaleza del crecimiento económico en Centroamérica.

El modelo utilizado para estimar la convergencia absoluta sigue la especificación propuesta por Baumol (1986), Barro \& Sala i Martin (1990), Bassanini, Scarpetta \& Hemmings (2001), el que se basa en estimar la magnitud del efecto del PIB per cápita de un estado inicial en la tasa de crecimiento a largo plazo de la economía.

$$
\log \left(\frac{y_{i, t+T}}{y_{i t}}\right) / T=\alpha+\beta \log \left(y_{i t}\right)
$$

Para probar la hipótesis de convergencia se define lo siguiente:

- Hipótesis nula: $\beta<0$ Convergencia en el crecimiento.

- Hipótesis alternativa: $\beta \geq 0$ Divergencia en el crecimiento.

El valor negativo del parámetro $\beta$ implica que los países con un PIB per cápita menor en estado inicial tenderían a crecer a una tasa más acelerada, por lo tanto en algún punto en el tiempo alcanzarían a los países de ingreso alto (lo que confirmaría la hipótesis de convergencia beta).

Según De Gregorio \& Lee (2003) se estimará una especificación alternativa, en donde el crecimiento económico estará en función de la diferencia entre el PIB per cápita del país con respecto al de la región. Las hipótesis por probar y la interpretación de esta ecuación son idénticas a la de la ecuación anterior, la ventaja de la ecuación propuesta por Gregorio es que resulta mucho más intuitiva, además de que supone convergencia a una tendencia común.

$$
\text { (4) } \quad \log \left(\frac{y_{i, t+T}}{y_{i t}}\right)=\alpha+\beta \log \left(\frac{y_{i t}}{\overline{y_{t}}}\right)
$$

Además de los dos modelos definidos anteriormente, se estimarán ecuaciones alternativas con diversas variables exógenas con el fin de cuantificar el efecto que tiene sobre el crecimiento la educación, desigualdad, salud, formación bruta de capital, etc. Para dar una explicación, a través de las políticas implementadas en Centroamérica, a la convergencia o divergencia en el crecimiento económico.

\footnotetext{
6 "El modelo de efectos fijos es una especificación apropiada si se está enfocado en un grupo específico de $\mathrm{N}$ empresas, como IMM GE Westinghouse, etc. y la inferencia está restricta al comportamiento de estas empresas. Alternativamente, puede ser N países de la OCDE, o N Estados Americanos" (Badi, Baltagi, 2005, pág. 14) (Traducción Propia).

${ }^{7}$ Los errores robustos reducen el sesgo que la heterocedasticidad y la autocorrelación producen sobre los errores estándar de los parámetros.
}

6

Marianela Molina Hernández y Marco Vinicio Valerio Berrocal

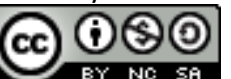

Revista Economía y Sociedad by Universidad Nacional is licensed under a Creative Commons Reconocimiento-NoComercialCompartirlgual 4.0 Internacional License. Creado a partir de la obra en http://www.revistas.una.ac.cr/index.php/economia 
Para estimar la convergencia relativa se calculará la dispersión entre niveles de PIB per cápita entre países, de manera que la convergencia relativa ocurre cuando la dispersión entre los niveles disminuye. La convergencia absoluta es una condición necesaria para que ocurra la convergencia relativa.

$$
\frac{\sigma_{\mathrm{t}+\mathrm{T}}}{\overline{\mathrm{X}}_{\mathrm{t}+\mathrm{T}}}<\frac{\sigma_{\mathrm{t}}}{\overline{\mathrm{X}}_{\mathrm{t}}}
$$

Los datos utilizados en este documento son obtenidos de la Comisión Económica para América Latina (CEPAL), estos son de periodicidad anual y abarcan la muestra de 1962 hasta 2012 y comprenden un corte transversal de los siguientes cinco países: Costa Rica, El Salvador, Nicaragua, Guatemala y Honduras. Los datos del PIB per cápita son armonizados por el método Atlas por el Banco Mundial, el cual “... aplica un factor de conversión que promedia el tipo de cambio de un año dado y los dos años anteriores, ajustados por diferencias en la tasas de inflación del país y, en el año 2000, los países del G5" (Banco Mundial, 2015), por lo tanto da una medida de ingreso ajustada por poder adquisitivo.

\section{Resultados}

En esta sección se detallan los resultados obtenidos a través del análisis descriptivo e inferencial de los datos. El análisis se centra en probar la hipótesis de convergencia, para ello se utilizan regresiones de datos de panel con distintas especificaciones y en distintas muestras, así como gráficos de las series de ingreso per cápita y volatilidades.

\subsection{Convergencia absoluta y relativa}

La figura 1 contiene las series de PIB per cápita para Centroamérica, a partir de 1983 se puede apreciar como Costa Rica comienza a crecer a tasas mayores que el resto de países de la región y comienza a divergir, esto debido a su no participación directa en el conflicto civil nicaragüense y el conflicto centroamericano. Por su parte, los países Guatemala, Salvador, Nicaragua y Honduras crecen todos a una tasa similar y en distintos periodos convergen entre ellos, estos se diferencian de Costa Rica, principalmente, en el sentido que tardan al menos una década en recuperar su ritmo de crecimiento, luego de la crisis de la balanza de pagos de los años 80.

A través de la muestra se identifican tres periodos de convergencia relativa entre los países, estos son: 1962-1967, 1979-1982 y 1990-2003. En el primer periodo que comprende de 1962 a1967 se atribuye al éxito relativo que tenía el modelo de desarrollo de la época que se basaba en la sustitución de importaciones, el Mercado Común Centroamericano y otras iniciativas de política económica. En el segundo periodo identificado que comprende de 1979 a 1982, la convergencia se debe a que de manera traslapada, pero dentro de este rango temporal, los países centroamericanos entran en crisis económica, y el país con mayor ingreso per cápita de la región, Costa Rica, es impactado en mayor medida que el resto de países, hasta que llega al año

Marianela Molina Hernández y Marco Vinicio Valerio Berrocal

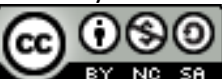

Revista Economía y Sociedad by Universidad Nacional is licensed under a Creative Commons Reconocimiento-NoComercialCompartirlgual 4.0 Internacional License. Creado a partir de la obra en http://www.revistas.una.ac.cr/index.php/economia 
1983 a alcanzar el mismo nivel de PIB per cápita que Guatemala. En el tercer periodo que comprende de 1990 a 2003 se le atribuirá (por el momento) la convergencia a los esfuerzos por una mayor integración económica, la globalización y al auge del comercio internacional.
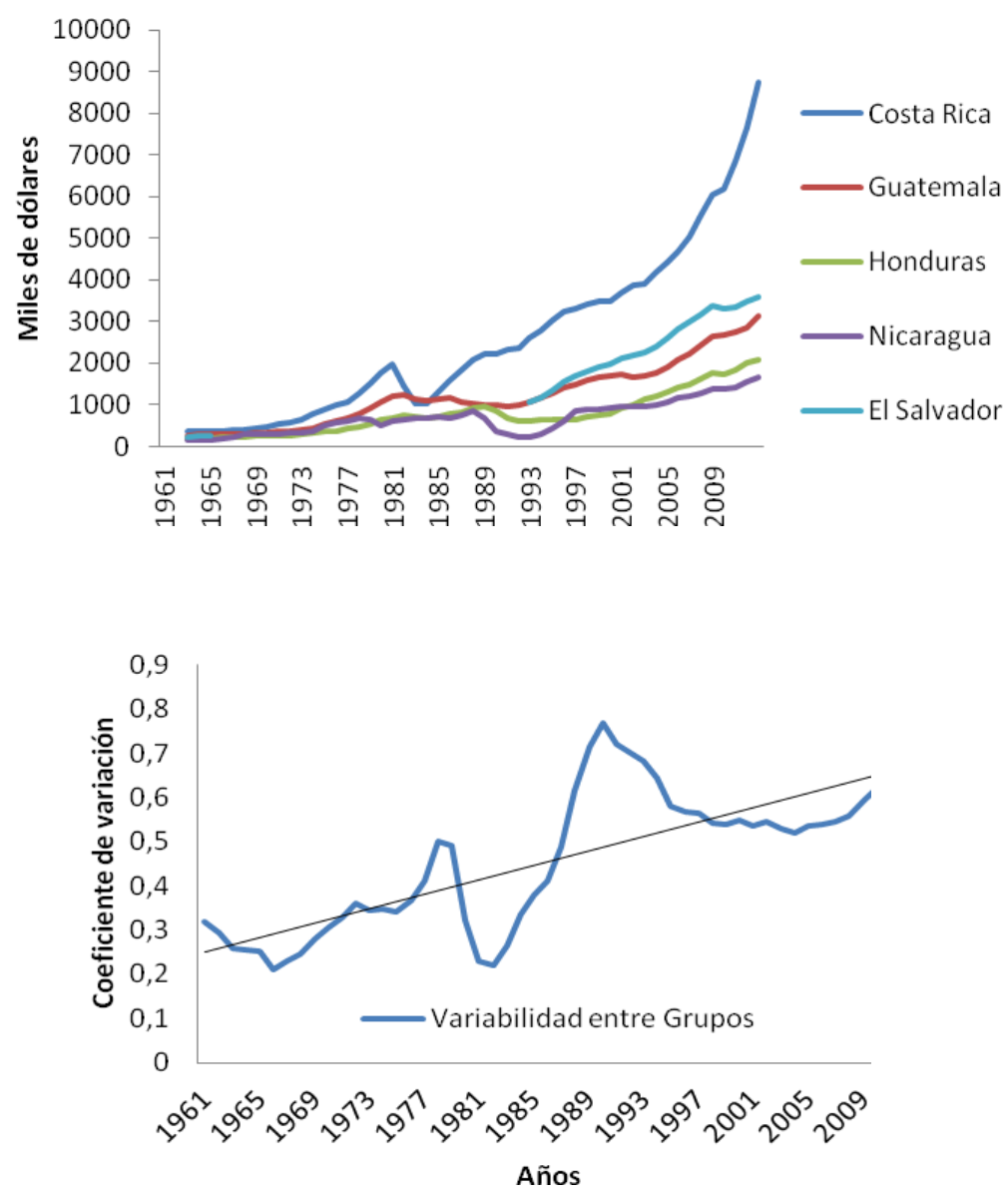

Figura 1. Centroamérica: producto interno bruto per cápita: Método Atlas y variabilidad entre grupos. Fuente: elaboración propia con base en datos del Banco Mundial, 2015.

Asimismo, es claro que entre los países existieron diferencias en materia de política económica que marcaron el desarrollo histórico de sus economías, y es atribuible a estas diferencias las distintas divergencias observadas en el crecimiento económico. En una óptica más amplia y en el marco de los modelos de crecimiento endógeno se pueden identificar otros causantes del crecimiento que vienen a explicar el "éxito" o "fracaso" de las economías. Algunos de estos factores son resultado de políticas públicas en educación, salud, redistribución del ingreso, etc.

8

Marianela Molina Hernández y Marco Vinicio Valerio Berrocal

(c) (i)(2)

Revista Economía y Sociedad by Universidad Nacional is licensed under a Creative Commons Reconocimiento-NoComercial- 
Los resultados de las estimaciones de la convergencia absoluta (beta) se presentan en la tabla 1 , como se mencionó en el apartado metodológico el coeficiente asociado al logaritmo del PIB per cápita mide la convergencia del crecimiento entre los países. El valor estimado para el periodo que comprende de 1962 al 2012 revela que existe evidencia a favor de la convergencia entre los países, pero no hay que apresurarse en hacer conclusiones; puesto que, a pesar de ser negativo el coeficiente es de una magnitud muy baja $(0,959 \%)$, por lo tanto, la convergencia tardaría siglos en completarse ${ }^{8}$. La teoría del crecimiento predice que la velocidad de convergencia está relacionada con el componente de capital físico contenido en la función de producción ${ }^{9}$, por lo que la baja velocidad de convergencia es evidencia de economías con una baja productividad marginal del capital, la evidencia encontrada por Kumo (2011) en los países del sur de África, los cuales presentan una velocidad de convergencia anual del 0,8\%, y estadísticamente no significativa. Por su parte, Goerlich, et al. (2005) calculan una velocidad de convergencia anual en los países europeos de una magnitud del 1,57\%.

Tabla 1.

Modelo: especificación de Barro Sala i Martin (1962-2012)

\begin{tabular}{ccc}
\hline \multicolumn{3}{c}{ Variable dependiente: } \\
\multicolumn{3}{c}{ crecimiento económico promedio de los últimos 10 años } \\
\hline Parámetro & Valor & P-value \\
\hline Logaritmo del PIB per cápita & $-0,09597$ & 0,00 \\
Intercepto & 0,7140 & 0,00 \\
\hline
\end{tabular}

Fuente: elaboración propia

La tabla 2 muestra la estimación de la especificación propuesta por De Gregorio \& Lee (2003) que está basada en brechas, es decir, se calculó la diferencia entre el valor de PIB observado para cada país y el promedio de la región.

Tabla 2.

Modelo: especificación de José De Gregorio (1960-2012)

\begin{tabular}{c|cc}
\multicolumn{3}{c}{ Variable dependiente: crecimiento económico promedio de los últimos 10 años } \\
\hline Parámetro & Valor & P-value \\
\hline Brecha & 0,0023 & 0,961 \\
Intercepto & 0,0037 & 0,743 \\
\hline
\end{tabular}

Fuente: elaboración propia

${ }^{8}$ En el anexo 1 se presentan los resultados de una simulación determinista del PIB per cápita.

${ }^{9}$ Véase Romer (2002) págs. 16-20

Marianela Molina Hernández y Marco Vinicio Valerio Berrocal

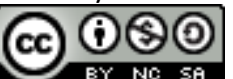

Revista Economía y Sociedad by Universidad Nacional is licensed under a Creative Commons Reconocimiento-NoComercial- 
La estimación provee evidencia en contra de la hipótesis de convergencia a una tendencia determinística común debido a que el signo del coeficiente beta es positivo y estadísticamente igual a cero. Para determinar si los países convergen a una tendencia estocástica común se aplicó una prueba de raíz unitaria de panel a la variable $\log \left(\mathrm{y}_{\mathrm{it}} / \overline{\mathrm{y}_{\mathrm{t}}}\right)$. La hipótesis nula es que existe convergencia, la hipótesis alternativa es que no existe convergencia a una tendencia estocástica común. La tabla con los resultados se presenta en el anexo 2 y ésta refleja que, además, se rechaza la hipótesis nula de convergencia a una tendencia estocástica común, esto implica que los países centroamericanos no tienden al PIB per cápita promedio de la región, sino que la convergencia es un Catch up los países de ingreso bajo tienden a alcanzar a los países de ingresos altos.

\subsection{Convergencia y determinantes del crecimiento}

Varias teorías económicas han tratado de explicar el fenómeno del crecimiento económico. En 1965, Robert Solow publica A Contribution to the Theory of Economic Growth en el que plantea una función de producción neoclásica agregada en la que el producto producido está en función de la acumulación de capital y del monto de mano de obra. Más adelante, autores como Romer (1986) y Lucas (1988) rompen con la corriente mainstream del crecimiento neoclásico e incorporan otros factores que pueden ser objeto de acumulación y que generan externalidades.

En esta sección se presenta una versión ampliada del modelo presentado en la tabla 1, esto con el fin de estimar la magnitud explicativa que tienen estas variables en el crecimiento a largo plazo. Ahora se incluirán variables relativas a la salud, educación, apertura comercial, política monetaria y desigualdad. Esta forma de estimación del parámetro de convergencia también es llamada "Convergencia condicional".

La variable que aproxima el nivel de educación de los países es el porcentaje de personas entre 15 y 19 años con primaria completa; la variable que aproxima el nivel de servicios de salud de cada país es el número de médicos por cada habitante; para medir el grado de apertura se utiliza el índice de apertura comercial que se define como la suma de importaciones y exportaciones dividida entre el producto interno bruto; como medida de desigualdad del ingreso se utiliza el índice de concentración de Gini, y para medir el efecto de la política monetaria se utiliza la inflación medida como la variación anual porcentual del índice de precios al consumidor.

10

Marianela Molina Hernández y Marco Vinicio Valerio Berrocal

(c) (i) (2)

Revista Economía y Sociedad by Universidad Nacional is licensed under a Creative Commons Reconocimiento-NoComercialCompartirlgual 4.0 Internacional License.

Creado a partir de la obra en http://www.revistas.una.ac.cr/index.php/economia 
Tabla 3.

Modelo: especificación Barro Sala i Martin, con otras variables explicativas (1960-2012)

Variable dependiente: crecimiento económico promedio de los últimos 10 años

\begin{tabular}{c|cc}
\hline Parámetro & Valor & P-value \\
\hline Logaritmo del PIB per cápita & $-0,0941$ & 0,00 \\
Logaritmo educación & 0,0665 & 0,00 \\
Inflación & 0,0025 & 0,226 \\
Logaritmo habitante por C/A médico & $-0,0346$ & 0,00 \\
Logaritmo del coeficiente de Gini & 0,1232 & 0,629 \\
Logaritmo índice de apertura comercial & 0,0179 & 0,002 \\
Intercepto & 0,4640 & 0,00 \\
\hline
\end{tabular}

Fuente: elaboración propia.

El resultado de la estimación se presenta en la tabla 3 , el valor que estima la convergencia resulta significativo con una probabilidad del $5 \%$ y revela una velocidad de convergencia estadísticamente igual. La estimación muestra que la variable proxy de educación es significativa, además su elasticidad indica que un incremento en el porcentaje de personas de 15 a 19 años con educación primaria completa de 1 punto porcentual (p.p) aumenta el crecimiento económico en 0,19 p.p. La apertura comercial y el aumento de médicos por habitante son significativas y tienen un impacto positivo sobre el crecimiento económico. Asimismo, las variables inflación y coeficiente de Gini no son significativas, con la utilización del test de causalidad de Granger para datos de panel no se puede definir una clara causalidad entre ambas variables (coeficiente de Gini y crecimiento económico a largo plazo; promedio de 10 años), pero a través de regresiones se encuentra una relación positiva significativa entre el crecimiento económico a corto plazo y la desigualdad en el ingreso, que sigue siendo positiva y de mayor magnitud a largo plazo $(0,35$ y 0,45 respectivamente).

\subsection{Proceso de liberalización y convergencia}

La tabla 4 muestra los resultados de la estimación del modelo de Barro \& Sala i Martin con la salvedad que en estas estimaciones la muestra es particionada en dos distintas sub muestras, la primera comprende el periodo de 1990-2012 y la segunda muestra comprende de 1960-1980. Con esta partición se pretende controlar el evento ocurrido en los años 80 de la crisis de la balanza de pagos y posterior proceso de ajuste estructural vivido por los países centroamericanos. La hipótesis nula es que a partir del proceso de liberalización en las economías la velocidad de convergencia debió acelerarse; debido a la mayor movilidad de factores productivos y el arbitraje. Además "el análisis de la convergencia macroeconómica debe por tanto, servir como una señal del grado de éxito de la estrategia de promoción de la integración" (Kumo, 2011). 
Tabla 4.

Modelo: especificación de Barro \& Sala i Martin, por periodos; 1960-1980/1990-2012

\begin{tabular}{|c|c|c|}
\hline \multicolumn{3}{|c|}{ Muestra: 1990-2012 } \\
\hline \multicolumn{3}{|c|}{$\begin{array}{l}\text { Variable dependiente: crecimiento económico promedio de los últimos } 10 \\
\text { años }\end{array}$} \\
\hline Parámetro & Valor & P-value \\
\hline Logaritmo del PIB per cápita & $-0,0765$ & 0,00 \\
\hline Intercepto & 0,5812 & 0,00 \\
\hline \multicolumn{3}{|c|}{ Muestra 1960-1980 } \\
\hline \multicolumn{3}{|c|}{$\begin{array}{l}\text { Variable dependiente: crecimiento económico promedio de los últimos } 10 \\
\qquad \text { años }\end{array}$} \\
\hline Parámetro & Valor & P-value \\
\hline Logaritmo del PIB per cápita & $-0,1180$ & 0,00 \\
\hline Intercepto & 0,8643 & 0,00 \\
\hline
\end{tabular}

Fuente: elaboración propia

Los resultados de las estimaciones muestran que para el periodo 1990-2012 la velocidad de convergencia es menor, es decir, se tardan más años en cerrar la brecha de crecimiento, por lo que la apertura comercial no favorece la convergencia como predice la teoría. Esto puede estar influenciado por la divergencia relativa identificada a partir del 2003. Si se excluye a Costa Rica y se recalcula la serie de dispersión entre PIB per cápita se encuentra que ahora solo existe una divergencia entre 1887-1991 (la década perdida), por lo que se puede decir que la divergencia vista a partir del 2003 es causada por Costa Rica que crece a tasas mayores que la región. Pero aún si se excluye a Costa Rica de las estimaciones, las velocidades de convergencia son menores en los años posteriores a 1990.

¿Por qué sucede esto? Las diferentes políticas económicas implementadas en los países centroamericanos son los causantes de esta desaceleración en el ritmo de convergencia, además existieron otros factores coyunturales que atravesaron algunas de estas economías como las catástrofes naturales ocurridas en Nicaragua y la guerra en Centroamérica que provocaron que para estos países la década de los 80 no fueran una "década perdida", sino que ocurrieron perdidas sostenidas en el crecimiento durante dos décadas. Asimismo, aunque Costa Rica comparte su historia y relaciones económicas con los otros países centroamericanos ha logrado distinguirse de estos en materia política, social y económica, en donde se muestra por ejemplo, mejores índices de desarrollo humano, de esperanza de vida, entre otros; gracias a la gran inversión en educación y salud que se realizó. Asimismo, las ayudas económicas de la

12

Marianela Molina Hernández y Marco Vinicio Valerio Berrocal

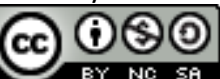

Revista Economía y Sociedad by Universidad Nacional is licensed under a Creative Commons Reconocimiento-NoComercialCompartirlgual 4.0 Internacional License. Creado a partir de la obra en http://www.revistas.una.ac.cr/index.php/economia 
Agencia Norteamericana para el Desarrollo Internacional (AID), a manera de transferencias, provocaron que la economía costarricense creciera a tasas superiores que la región.

\section{4. ¿Convergencia en las tasas de crecimiento?}

Una pregunta que queda por hacerse es la siguiente: ¿la desaceleración del ritmo de la convergencia en el PIB per cápita es un resultado de una convergencia en las tasas de crecimiento? La convergencia en las tasas de crecimiento implica que los países de ingreso bajo nunca alcanzarían a los países de ingreso alto. Para probar esta hipótesis se calculó la dispersión entre estas tasas de crecimiento, una menor dispersión implica que estas tasas son en el tiempo cada vez más similares y por lo tanto convergen.

La figura 2 muestra el desvío estándar entre tasas de crecimiento. Esta dispersión muestra una tendencia decreciente a través del tiempo, lo que es evidencia a favor de la convergencia en las tasas de crecimiento.

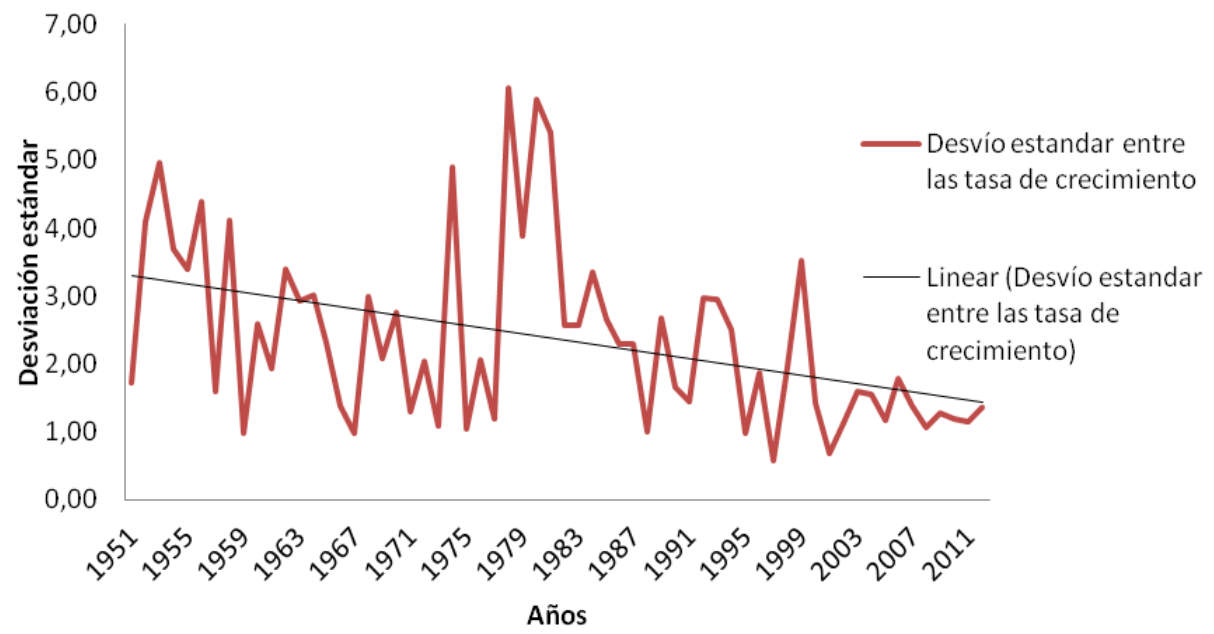

Figura 2. Centroamérica: dispersión entre tasas de crecimiento.

Fuente: elaboración propia.

Esto explica en gran medida la desaceleración en la ya baja velocidad de convergencia en el PIB per cápita de los países centroamericanos, puesto que si los países tienden a igualar sus tasas de crecimiento nunca lograrían niveles PIB per cápita y bienestar idénticos.

\subsection{Desigualdad y crecimiento}

Como último apartado de la sección de resultados se estudia los efectos cuantitativos del crecimiento económico y otras variables relacionadas con salud, educación, política monetaria y 
comercio, sobre la desigualdad del ingreso, medida por el índice de concentración de Gini. La tabla 5 muestra el resultado de las estimaciones.

Tabla 5.

Modelo: relación de desigualdad (Coef. GINI) y tasa de crecimiento

Muestra: 1990-2012

Variable dependiente: Log. Coef. Gini

\begin{tabular}{c|cc}
\hline Parámetro & Valor & P-value \\
\hline Crecimiento & 0,3547 & 0,052 \\
Educación & $-0,1044$ & 0,010 \\
Inflación & $-0,0067$ & 0,623 \\
Habitantes por C/A médico & $-0,0240$ & 0,061 \\
Índice de apertura comercial & $-0,1280$ & 0,000 \\
Intercepto & 0,5494 & 0,006 \\
\hline
\end{tabular}

Fuente: elaboración propia

Los resultados de la estimación revelan que existe trade off entre crecimiento y desigualdad en las economías de Centroamérica, este resultado es congruente con la hipótesis de Kuznets (1955). La hipótesis de Kuznets consiste en que en etapas tempranas del desarrollo la desigualdad del ingreso aumenta y tiende a decaer en etapas posteriores, por lo que en economías en vías de desarrollo es de esperarse una relación positiva entre el coeficiente de Gini y el crecimiento económico. En Centroamérica se estima que un incremento del crecimiento económico de 1 p.p. genera un aumento de 0,35\% en el coeficiente de concentración de Gini. Esto quiere decir que existe evidencia en contra de la teoría del "Goteo" que parece no cumplirse en las economías centroamericanas.

La apertura comercial y la educación tienden a tener un efecto mayor en la reducción de la desigualdad del ingreso. La apertura comercial posee un efecto negativo en la desigualdad, esto quiere decir que se cumplen condiciones de arbitraje, por lo que los salarios mantendrán una tendencia a igualarse entre los países centroamericanos. La educación tiene un gran efecto en la reducción de la desigualdad, la educación es una fuente de oportunidades para la población y permite a la población alcanzar un mayor ingreso, este efecto se estima en una reducción del $10 \%$ del coeficiente de Gini por cada punto porcentual en que se incrementa el porcentaje de graduados de primaria ${ }^{10}$.

10 En regresiones adicionales no reportadas se determina que el efecto en el incremento de los graduados de segundaria es similar, alrededor de un $10 \%$ sobre el coeficiente de Gini.

14

Marianela Molina Hernández y Marco Vinicio Valerio Berrocal

(c) (1) 8 (2)

Revista Economía y Sociedad by Universidad Nacional is licensed under a Creative Commons Reconocimiento-NoComercialCompartirlgual 4.0 Internacional License. Creado a partir de la obra en http://www.revistas.una.ac.cr/index.php/economia 
Por último, la política monetaria tiene un efecto negativo y no significativo en el coeficiente de Gini; lo que indica que no es una herramienta efectiva para reducir la desigualdad del ingreso, o bien, los ingresos por impuesto inflacionario no tienen un impacto significativo en la desigualdad.

\section{Conclusiones}

En este documento se identificó que el proceso de convergencia se da a cabo a una velocidad muy baja, equivalente a un $0,9597 \%{ }^{11}$ anual, y que esta disminuyó durante de la aplicación de los programas de ajuste estructural, y tiende a disminuir en la actualidad.

El proceso de convergencia es un catch up, lo que quiere decir que los países de ingreso bajo tratan de alcanzar a los países de ingreso alto, y no en es una convergencia a una tendencia común (estocástica o determinística).

Adicionalmente, se halló que las variables de educación, salud y apertura comercial tienen un aporte significativo en el crecimiento a largo plazo, además mantener inflaciones bajas en los países centroamericanos ha propiciado mayores tasas de crecimiento.

También, se determinó que existe un trade off entre crecimiento y desigualdad, a mayores tasas de crecimiento se encuentran mayores índices de Gini lo que indica mayor desigual, lo que es consistente con la hipótesis de Kuznets (1955).

Por último, la convergencia entre los países se da en las tasas de crecimiento, por lo que la convergencia de los PIB per cápita puede nunca darse a cabo. Todos estos hallazgos indican que una integración regional económica puede no ser viable debido a que las diferencias entre las economías de la región son muy marcadas y no existe una velocidad de convergencia alta.

${ }^{11}$ La duración mediana de convergencia $\frac{\log (2)}{-\beta}$ para un $\beta_{\text {anual }}=-0.0095$ es 72 años. 


\section{Referencias}

Antunez, C. (2011). Crecimiento Económico: Modelos de Crecimiento Económico. Recuperado de http://www.eumed.net/librosgratis/2010d/761/Que\%20causa\%20el\%20Crecimiento\%2 OEconomico.htm

Baltagi, B. (2005). Econometric Analysis of Panel Data. England: John Wiley \& and Sons. Retrieved from https://books.google.co.cr/books/about/Econometric Analysis of Cross Section an.ht $\mathrm{ml}$ ?id=cdBPOJUP4VsC\&redir esc $=y$

Banco Mundial (2015). El método Atlas del Banco Mundial. Recuperado de http://datos.bancomundial.org/quienes-somos/clasificacion-paises/atlas

Barro, R., \& Sala-i-Martin , X. (1990). Economic growth and convergence across the United States (Working Paper No. 3419). Retrieved from University of Massachusetts, the National Bureau of economic research website: http://www.nber.org/papers/w3419

Bassanini, A., Scarpetta, S., \& Hemmings, P. (2001). Economic growth: The role of policies and intitutions: Panel data. Evidence from OECD Countries (No 283) . Retrieved from Economics Department Working Papers website: http://www.oecdilibrary.org/economics/economic-growth-the-role-of-policies-andinstitutions 722675213381

Baumol, W. (1986). Productivity Growth, Convergence, and welfare: What the long run data show. The American Economic Review, 76(5),1072-1085. Recuperado de http://piketty.pse.ens.fr/files/Baumol1986.pdf

De Gregorio, J., \& Lee, J.-W. (2003). Growth and adjustment in East Asia and Latin America (No 245). Recuperado del sitio del Banco Central de Chile: http://www.bcentral.cl/estudios/documentos-trabajo/fichas/245.htm

Goerlich, F. J., Mas, M., \& Pérez , F. (2005). Crecimiento y convergencia en la Unión Europea. En Jordan, J. (Ed. Thomson-Civitas). Economía de la Unión Europea. Recuperado de http://www.uv.es/perezgar/publicaciones/CrecimientoUE.pdf

Kumo, W. (2011). Growth and macroeconomic convergence in Southern Africa (Series No 130). Retrieved from African Development Bank Group Working Paper website: http://www.afdb.org/fileadmin/uploads/afdb/Documents/Publications/WORKING\%201 30\%20GROWTH\%20AND\%20MACROECONOMIC\%20CONVERGENCE\%20.pdf

16

Marianela Molina Hernández y Marco Vinicio Valerio Berrocal

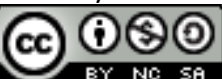

Revista Economía y Sociedad by Universidad Nacional is licensed under a Creative Commons Reconocimiento-NoComercialCompartirlgual 4.0 Internacional License. Creado a partir de la obra en http://www.revistas.una.ac.cr/index.php/economia 
Kuznets, S. (1955). Economic growth and Income inequality. The American Economic Review. Vol 1 (XLV), 1-28. Recuperado de https://www.aeaweb.org/aer/top20/45.1.1-28.pdf

Lucas. R. (1988). On the mechanics of economic develoment. Journal of monetary economics, 1 (22), pp 3-42. doi:10.1016/0304-3932(88)90168-7

Romer, D. (2002). Macroeconomía avanzada. Recuperado de http://www.mheducation.com.mx/9788448148096-latam-macroeconomia-avanzada-3e

Romer, P. (1986). Increasing Returns and Long-Run Growth. The Journal of Political Economy, 5(94), pp 1002-1037. $\quad$ Recuperado de http://www.jstor.org/stable/1833190?seq=1\#page scan tab contents 


\section{Anexos}

\section{Anexo 1:}

\section{Pronósticos}

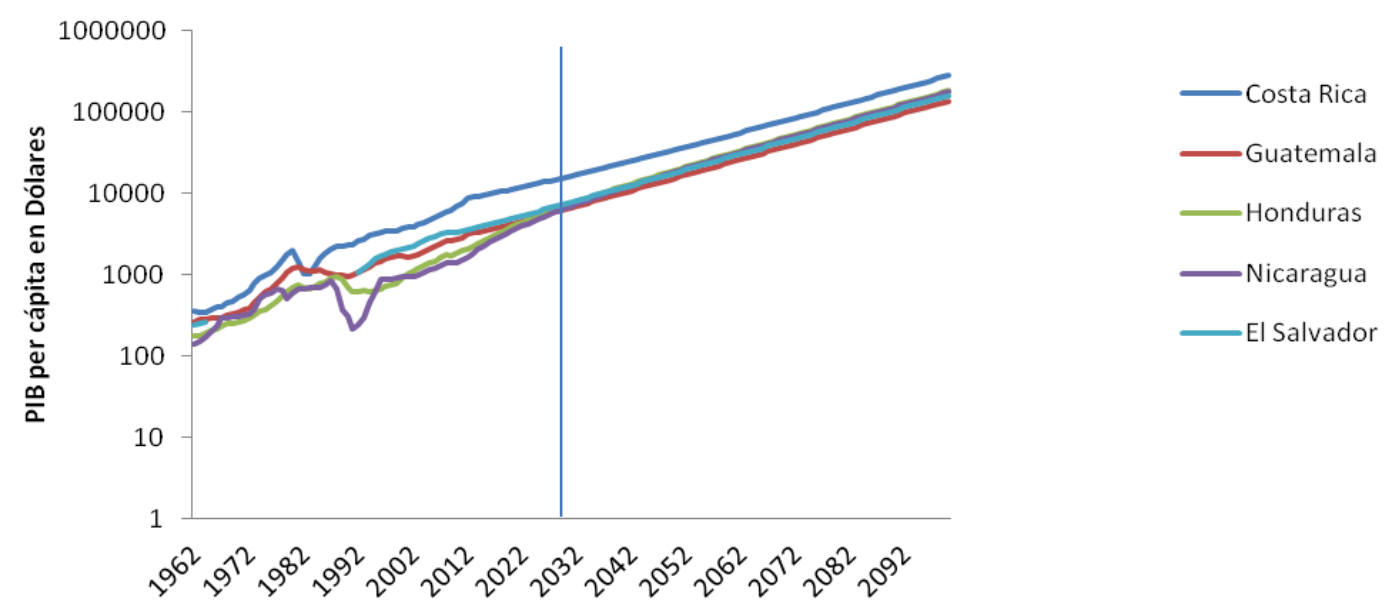

Figura 3. Centroamerica: Simulación de convergencia $\beta=-0,0997$. Fuente: Elaboración propia 
Anexo 2:

\section{Prueba de Raíz Unitaria de panel}

Levin-Lin-Chu unit-root test for $\log \left(y_{i t} / \bar{y}_{t}\right)$,

\begin{tabular}{ll}
\hline Ho: Panels contain unit roots & Number of panels $=5$ \\
\hline Ha: Panels are stationary & Number of periods $=20$ \\
\hline AR parameter: Common & Asymptotics: N/T $->0$ \\
\hline
\end{tabular}

Panel means: Included

Time trend: Not included

ADF regressions: 1 lag

LR variance: Bartlett kernel, 12.00 lags average (chosen by LLC)

\begin{tabular}{lll}
\hline & Statistic & p-value \\
\hline Unadjusted t & -3.8834 & \\
Adjusted t* & -1.8624 & $\mathbf{0 . 0 3 1 3 ^ { * }}$
\end{tabular}

$\overline{\text { Fuente: elaboración propia con base en estimaciones realizadas }}$

Anexo 3:

Prueba Causalidad de Granger

Pairwise Granger Causality Tests

Lags: 2

\begin{tabular}{lccc}
\hline \hline Null Hypothesis: & Obs & F-Statistic & Prob. \\
& & & \\
\hline \hline Crecimiento does not Granger Cause GINI & 105 & 0.50827 & 0.6031 \\
GINI does not Granger Cause Crecimiento & & 1.15452 & 0.3194
\end{tabular}

Fuente: elaboración propia con base en estimaciones realizadas 